ThE Astrophysical Journal, 369:L9-L12, 1991 March 1

(C) 1991. The American Astronomical Society. All rights reserved. Printed in U.S.A.

\title{
ELECTRON DENSITIES AND THE EXCITATION OF CN IN MOLECULAR CLOUDS
}

\author{
JoHn H. BLACK ${ }^{1,2}$ AND EWINE F. VAN DishoECK ${ }^{3,4}$ \\ Received 1990 September 19; accepted 1990 December 5
}

\begin{abstract}
In molecular clouds of modest density and relatively high fractional ionization $\left[n(e) / n\left(\mathrm{H}_{2}\right)>10^{-5}\right]$, the rotational excitation of $\mathrm{CN}$ is controlled by a competition among electron impact, neutral impact (primarily $\mathrm{H}_{2}$ ), and the interaction with the cosmic background radiation. The degree of excitation can be measured through optical absorption lines and millimeter-wave emission lines. The available, accurate data on $\mathrm{CN}$ in diffuse and translucent molecular clouds are assembled and used to determine electron densities. The derived values, $n(e) \approx 0.02-0.5 \mathrm{~cm}^{-3}$, imply modest neutral densities in the range $10^{2.5}-10^{3.5} \mathrm{~cm}^{-3}$, which generally agree well with determinations by other techniques. The absorption- and emission-line measurements of $\mathrm{CN}$ both exclude densities higher than $n\left(\mathrm{H}_{2}\right) \approx 10^{3.5} \mathrm{~cm}^{-3}$ on scales varying from $10^{-3}$ to $60^{\prime \prime}$ in these clouds.
\end{abstract}

Subject headings: interstellar: matter — interstellar: molecules - molecular processes

\section{INTRODUCTION}

The small ionized fraction is an important property of predominantly neutral molecular clouds in the Galaxy. The positive ions play a major role in gas phase chemistry. It is the charged species that govern the coupling of the gas to magnetic fields: thus they help control the dynamical effects of fields on cloud structure and evolution. Measurements of ion densities or electron fractions can be used as remote probes of the fluxes of ultraviolet starlight and penetrating cosmic rays. In addition, the electron density affects the charge state of interstellar dust particles and thus partly dictates the efficiency of heating by the photoelectric effect and the ways in which the dust affects the chemistry. Finally, even at modest electron fractions, $n(e) / n\left(\mathbf{H}_{2}\right) \approx 10^{-5}$ to $10^{-4}$, the electron collisions can dominate the excitation of polar neutral molecules and molecular ions. This last point can be exploited, in favorable cases, to determine interstellar electron densities from observations.

Optical absorption-line measurements of $\mathrm{CN}$ in diffuse clouds have long been used to measure the brightness temperature of cosmic background radiation at the wavelengths of its two lowest rotational transitions, 2.6 and $1.3 \mathrm{~mm}$ (Shklovsky 1966; Field \& Hitchcock 1966; Thaddeus \& Clauser 1966). Since the $C O B E$ satellite has firmly established that the background has a blackbody spectrum at a temperature $T_{\mathrm{bg}}=2.735 \pm 0.06 \mathrm{~K}$ (Mather et al. 1990), the recent $\mathrm{CN}$ absorption line observations of high signal-to-noise ratio and high resolution (Palazzi et al. 1990; Crane et al. 1986, 1989; Lambert, Sheffer, \& Crane 1990; Kaiser \& Wright 1990; Meyer et al. 1989) can now be used confidently to measure the rotational excitation of $\mathrm{CN}$ in excess of $T_{\mathrm{bg}}$. The $\mathrm{CN}$ molecule has a high dipole moment, $\mu=1.45 \mathrm{D}$ (Thompson \& Dalby 1968 ), and is thus very susceptible to excitation through electron impact (cf. Thaddeus 1972; Black 1988). The excess excitation reflects the density and temperature where the $\mathrm{CN}$ lies and therefore measures the electron density when the neutral density and temperature are constrained by other observa-

\footnotetext{
1 Onsala Space Observatory.

${ }^{2}$ Postal address: Steward Observatory, University of Arizona, Tucson, AZ 85721 .

${ }^{3}$ Division of Geological and Planetary Sciences, California Institute of Technology.

${ }^{4}$ Sterrewacht Leiden, P.O. Box 9513, 2300 RA Leiden, The Netherlands.
}

tions. It is now feasible to detect weak $\mathrm{CN}$ millimeter emission lines in diffuse and translucent clouds, where the line intensity can also be related to the electron density. The contribution of electron collisions to the local excitation of $\mathrm{CN}$ has been discussed previously by Thaddeus (1972) and Meyer \& Jura (1985).

Although the basic molecular data for $\mathrm{CN}$, including the cross sections for electron-impact excitation, are well determined, there is one serious deficiency: cross sections for excitation by collisions with $\mathrm{H}_{2}$ have never been calculated or measured.

\section{OBSERVATIONS OF INTERSTELLAR CN}

Measurements of the interstellar absorption lines in the violet system of $\mathrm{CN}, B^{2} \Sigma^{+}-X^{2} \Sigma^{+}$, can provide column densities in each of the lowest rotational levels, $N=0,1$, and 2 . The corresponding rotational excitation temperatures for pairs of states $N^{\prime}$ and $N^{\prime \prime}, T_{N^{\prime \prime} N^{\prime}}$, tend toward $T_{N^{\prime \prime} N^{\prime}} \sim T_{\mathrm{bg}}=2.735 \mathrm{~K}$ in the limit of low densities and toward $T_{N^{\prime \prime} N^{\prime}} \sim T$ in the limit of high densities, where $T$ without subscript is the kinetic temperature. Excepting the very recent observations of $\mathrm{CN}$ toward $\zeta$ Oph by Lambert et al. (1990), the optical absorption lines are usually unresolved and only equivalent widths, $W_{\lambda}=$ $\int\left[1-\exp \left(-\tau_{\lambda}\right)\right] d \lambda$, are measured. Even in relatively thin clouds, the optical depth may be large, $\tau_{\lambda} \gtrsim 1$, so that saturation complicates the determination of column densities from $W_{\lambda}$, particularly when the object is to measure a small difference between $T_{N^{\prime \prime} N^{\prime}}$ and $T_{\mathrm{bg}}$. Data of sufficiently high signal-tonoise ratio permit the $N=1$ column density and Doppler linebroadening parameter $b$ to be determined simultaneously from the equivalent widths of the $R(1)$ and $P(1)$ line blends that have a common initial state. Small values of $b \leq 0.5 \mathrm{~km} \mathrm{~s}^{-1}$ enhance the effects of the intrinsic blending of unresolved spin rotation components on the strengths of the observed absorption features. As a result, the observed column densities and rotational populations are usually sensitive to the value of $b$. The Doppler parameter can also be estimated from the widths of resolved millimeter-wave emission lines of other molecules like ${ }^{13} \mathrm{CO}$, observed in the same direction as the background star. Caution must be exercised: the projected antenna beam collects emission from molecules distributed over a much larger volume than that sampled by the line of sight directly to the star and may include molecules that lie behind the star. 
The other observational input is from rotational emission lines at millimeter wavelengths. Here, the Rayleigh-Jeans radiation temperature of the line (i.e., the antenna temperature of extended emission that would be measured by a perfectly efficient antenna) is

$$
T_{R}\left(F^{\prime}, F^{\prime \prime}\right)=\frac{c^{2}}{2 k v^{2}}\left\{\left[B_{v}\left(T_{F^{\prime \prime} F^{\prime}}\right)-B_{v}\left(T_{\mathrm{bg}}\right)\right]\left[1-\exp \left(-\tau_{v}\right)\right]\right\} \mathrm{K},
$$

where $B_{v}(T)$ is the Planck function for temperature $T$ evaluated at line center frequency $v$. The line center optical depth is

$$
\begin{aligned}
\tau_{v}= & 3.738 \times 10^{-7} \frac{c^{3} A_{F^{\prime} F^{\prime \prime}} N_{J^{\prime \prime} F^{\prime \prime}}\left(2 F^{\prime}+1\right)}{v^{3} \Delta V\left(2 F^{\prime \prime}+1\right)} \\
& \times\left[1-\exp \left(\frac{-h v}{k T_{F^{\prime \prime} F^{\prime}}}\right)\right]
\end{aligned}
$$

for a Gaussian line of full width at half-maximum, $\Delta V=1.665 b$ in $\mathrm{km} \mathrm{s}^{-1}$, when $c / v$ is in $\mathrm{cm}$. The spontaneous transition probability is $A_{F^{\prime} F^{\prime \prime}}\left(\mathrm{s}^{-1}\right)$ and the transitions are now designated by the quantum number including electron and nuclear spin, $F$, to signify that spin rotation and hyperfine structure is resolved in the millimeter wave observations. $N_{J^{\prime \prime} F^{\prime \prime}}$ is the column density of the molecule in the lower level in $\mathrm{cm}^{-2}$. In all that follows, we will specify measurements and predictions for the strongest of the spin-rotation-hyperfine components: $N=1 \rightarrow 0, \quad J=3 / 2 \rightarrow 1 / 2, \quad F=5 / 2 \rightarrow 3 / 2$ at $113.4909 \mathrm{GHz}$ and $N=2 \rightarrow 1, J=5 / 2 \rightarrow 3 / 2, F=7 / 2 \rightarrow 5 / 2$ at 226.8748 GHz (Skatrud et al. 1983).

In regions that show evidence of complicated velocity structure on scales comparable to the optical resolution, the multicomponent structure has been taken into account in the analysis.

\section{MOLECULAR PROCESSES AND ANALYSIS}

The equivalent widths of $\mathrm{CN}$ absorption lines have been analyzed by comparison with equivalent widths computed by direct integration over theoretical line profiles. The computed profiles represent each velocity component of each spinrotation fine-structure component by a Voigt function that is characterized by the oscillator strength, $f$, the Doppler parameter of that velocity component, $b$, and the inverse lifetime of the upper level, $A_{\text {tot }}=1.61 \times 10^{6} \mathrm{~s}^{-1}$ for $\mathrm{CN} B-X(0,0)$. The technique is similar to that described by Black and van Dishoeck (1988), except that the spin-rotation fine-structure components are included explicitly using the best available data on line positions (P. F. Bernath 1990, private communication). It yields the column densities in the various levels from which the excitation temperature $T_{01}$ can be determined.

In Table 1 we summarize the observed and derived properties $T_{01}, T_{R}(113.491), N(\mathrm{CN})$, and $b$, with references to the observations on which these are based. The published optical measurements have been reanalyzed, when necessary, for our adopted value of oscillator strength of the $\mathrm{CN} B-X(0,0)$ band, namely $f_{00}=0.034$ (Bauschlicher, Langhoff, \& Taylor 1988). Two pairs of values of $b$ and $N(C N)$ are tabulated: one, $b($ opt $)$, determined from the ratio of equivalent widths in the $R(1)$ and $P(1)$ absorption features and the other, $b\left({ }^{13} \mathrm{CO}\right)$, from the width of the ${ }^{13} \mathrm{CO} J=1 \rightarrow 0$ emission line in the same direction. Small disagreements can have significant consequences. For example, for the line of sight toward HD 169454, the measured $R(1) / P(1)$ equivalent width ratio indicates values of $b$ between 0.3 and 0.5 , which would imply a very large range of total column densities of $(0.3-2.7) \times 10^{14} \mathrm{~cm}^{-2}$. The corresponding range of excitation temperatures, $T_{01}=3.6-1.6 \mathrm{~K}$, includes unphysical values at the lower end. In such cases, $b\left({ }^{13} \mathrm{CO}\right)$ was adopted in the subsequent analysis.

The observed $T_{R}(113)$ and derived $T_{01}$ are compared with results of a non-LTE calculation of rotational excitation that incorporates the observed $b$ and $N(\mathrm{CN})$ and that treats the radiative transfer by the method of mean escape probabilities. Line frequencies and relative line strengths are from Skatrud et al. (1983), from which we have computed transition probabilities for a dipole moment $\mu=1.45 \mathrm{D}$. Rate coefficients for deexcitation by electron impact were adopted from Allison \& Dalgarno (1971) and supplemented for higher levels through use of the formula of Dickinson et al. (1977). Rate coefficients for the different spin-hyperfine components are taken to scale proportionally to the radiative line strengths. Up to 65 transitions between 25 levels of $N=0,1,2,3$, and 4 were included in the calculation. For lack of anything better, we have applied the $\mathrm{H}_{2}-\mathrm{CS}$ collisional deexcitation rates of Green \& Chapman (1978) to the $\mathrm{H}_{2}-\mathrm{CN}$ system. In contrast to $\mathrm{CN}\left(X^{2} \Sigma^{+}\right), \mathrm{CS}$ $\left(X^{1} \Sigma^{+}\right)$has a dipole moment that is $35 \%$ larger and a rotational constant that is approximately a factor of 2 smaller. Also, because $\mathrm{CN}$ has nonzero spin, the interactions with ortho- and para- $\mathrm{H}_{2}$ are likely to be different. Even so, the typical rates may not be in error by more than factors of 2-3. The excitation calculation yields non-LTE populations of spin-rotation-hyperfine levels, from which $T_{01}$ and $T_{12}$ are computed by summing over spin-hyperfine sublevels.

To illustrate the competition among radiative and collisional processes, we can compare rates for the $N=0 \rightarrow 1$ $J=1 / 2 \rightarrow 3 / 2$ transition, averaged over the hyperfine structure: the rate of absorption of $2.735 \mathrm{~K}$ background radiation is $1.5 \times 10^{-7} \mathrm{~s}^{-1}$; the rate of excitation by electron collisions at $T=20 \mathrm{~K}$ is $4.3 \times 10^{-7} n(e) \mathrm{s}^{-1}$ and by $\mathrm{H}_{2}$ collisions is 3.8 $\times 10^{-11} n\left(\mathrm{H}_{2}\right) \mathrm{s}^{-1}$. This indicates that for $n(e) / n\left(\mathrm{H}_{2}\right) \gtrsim 10^{-4}$, electron impact competes effectively with neutral impact, and for $n(e) \geq 0.1 \mathrm{~cm}^{-3}$, some noticeable excitation, $T_{01}>T_{\mathrm{bg}}$, is expected.

\section{RESULTS}

The calculation of rotational excitation in $\mathrm{CN}$ takes as input the column densities and line widths determined from optical observations and uses values of the kinetic temperature inferred from other observations. Where no information on $T$ is available, a value $T=25 \mathrm{~K}$ has been assumed. The excitation calculation is performed for a range of values of $n(e)$ and $n(e) / n\left(\mathrm{H}_{2}\right)$ and yields values of $T_{N^{\prime \prime} N^{\prime}}$ and $T_{R}$ for all transitions. Comparison with measurements of or limits on $T_{01}$ and $T_{R}$ then provides values of $n(e)$. The results of this analysis are presented in Table 1 . For some clouds, notably toward $\zeta$ Per, $\zeta$ Oph, and HD 169454, detailed models of a variety of observations specify probable values of the electron fraction (van Dishoeck \& Black 1986; Lepp et al. 1988; Jannuzi et al. 1988), especially in cases where the ultraviolet lines of atomic species like $\mathrm{C}$ and $\mathrm{C}^{+}$have been measured. For other clouds, a plausible range of $n(e) / n\left(\mathrm{H}_{2}\right) \approx 3 \times 10^{-5}$ to $3 \times 10^{-4}$ has been adopted (see below). The derived electron densities and neutral densities are listed in the eleventh through fourteenth columns, first as constrained by the measured values of $T_{01}$ and second as indicated by observed limits on $T_{R}(113.49)$. The results in Table 1 refer to the nominal values of the observed properties, without consideration of the uncertainties. For comparison, Table 1 includes the neutral densities determined from other 
TABLE 1

Observed Properties and Derived Electron Densities

\begin{tabular}{|c|c|c|c|c|c|c|c|c|c|c|c|c|c|c|c|}
\hline \multirow[b]{2}{*}{ Star } & \multicolumn{7}{|c|}{ Observed Properties } & \multicolumn{2}{|c|}{ Adopted } & \multicolumn{4}{|c|}{ Derived properties } & \multicolumn{2}{|c|}{ Comparison } \\
\hline & $\begin{array}{l}T_{01} \\
(\mathrm{~K})\end{array}$ & $\begin{array}{l}b(\mathrm{opt}) \\
\left(\mathrm{km} \mathrm{s}^{-1}\right)\end{array}$ & $\begin{array}{l}N(\mathrm{CN}) \\
\left(\mathrm{cm}^{-2}\right)\end{array}$ & $\begin{array}{l}T_{R}(113) \\
(\mathrm{mK})\end{array}$ & $\begin{array}{l}b\left({ }^{13} \mathrm{CO}\right) \\
\left(\mathrm{km} \mathrm{s}^{-1}\right)\end{array}$ & $\begin{array}{l}N(\mathrm{CN}) \\
\left(\mathrm{cm}^{-2}\right)\end{array}$ & Ref. & $\begin{array}{l}T^{a} \\
(\mathrm{~K})\end{array}$ & $\frac{n\left(\mathrm{e}^{6}\right.}{n\left(\mathrm{H}_{2}\right)}$ & $\begin{array}{l}n(\mathrm{e})^{c} \\
\left(\mathrm{~cm}^{-3}\right)\end{array}$ & $\begin{array}{l}n\left(\mathrm{H}_{2}\right)^{c} \\
\left(\mathrm{~cm}^{-3}\right)\end{array}$ & $\begin{array}{l}n(\mathrm{e})^{d} \\
\left(\mathrm{~cm}^{-3}\right)\end{array}$ & $\begin{array}{l}n\left(\mathrm{H}_{2}\right)^{d} \\
\left(\mathrm{~cm}^{-3}\right)\end{array}$ & $\begin{array}{l}n\left(\mathrm{H}_{2}\right)^{e} \\
\left(\mathrm{~cm}^{-3}\right)\end{array}$ & $\begin{array}{l}n\left(\mathrm{H}_{2}\right)^{f} \\
\left(\mathrm{~cm}^{-3}\right)\end{array}$ \\
\hline HD 21483 & $2.81 \pm .03$ & 1.30 & $3.65(13)$ & $<20$ & 0.70 & ... & 1,2 & 25 & $\begin{array}{l}3.3(-4) \\
1.0(-4) \\
3.3(-5)\end{array}$ & $\begin{array}{l}0.10 \\
0.063 \\
0.031\end{array}$ & $\begin{array}{l}300 \\
630 \\
930\end{array}$ & $\begin{array}{l}<0.085 \\
<0.049 \\
<0.022\end{array}$ & $\begin{array}{l}<255 \\
<490 \\
<660\end{array}$ & ... & $300-7000$ \\
\hline$\zeta$ Per & $2.79 \pm .03$ & $1.25 \pm .02$ & $3.20(12)$ & $\cdots$ & $\ldots$ & $\ldots$ & 3 & 30 & $3.6(-4)$ & 0.075 & 210 & $\cdots$ & $\cdots$ & 150 & $\cdots$ \\
\hline HD 26571 & $3.4_{-.6}^{+.8}$ & 3. & $1.05(13)$ & $<25$ & 0.72 & $1.51(13)$ & $2,4,6$ & 25 & $1.0(-4)$ & 0.59 & 5900 & $<0.08$ & $<800$ & $\ldots$ & $500-5000$ \\
\hline $\mathrm{HD} 62542^{g}$ & $3.3 \pm .2$ & 0.69 & $2.96(13)$ & $\ldots$ & 0.66 & $3.17(13)$ & 2,5 & 55 & $\begin{array}{l}3.3(-4) \\
1.0(-4) \\
3.3(-5)\end{array}$ & $\begin{array}{l}0.49 \\
0.29 \\
0.13\end{array}$ & $\begin{array}{l}1460 \\
2850 \\
3915\end{array}$ & $\begin{array}{l}\cdots \\
\cdots \\
\cdots\end{array}$ & $\begin{array}{l}\cdots \\
\cdots \\
\ldots\end{array}$ & $\geq 300$ & $500-3000$ \\
\hline HD $62542^{h}$ & $2.8 \pm .4$ & 0.72 & $4.0(13)$ & $70 \pm 15$ & 0.66 & $4.4(13)$ & 6 & 55 & $\begin{array}{l}3.3(-4) \\
1.0(-4) \\
3.3(-5)\end{array}$ & $\begin{array}{l}0.047 \\
0.025 \\
0.011\end{array}$ & $\begin{array}{l}140 \\
250 \\
330\end{array}$ & $\begin{array}{l}0.13 \\
0.06 \\
0.025\end{array}$ & $\begin{array}{l}390 \\
600 \\
750\end{array}$ & $\geq 300$ & $500-3000$ \\
\hline HD 80077 & $2.9 \pm .2$ & $\ldots$ & $\ldots$ & $<25$ & 0.66 & $2.97(13)$ & 2,6 & 25 & $\begin{array}{l}3.3(-4) \\
1.0(-4)\end{array}$ & $\begin{array}{l}0.25 \\
0.16\end{array}$ & $\begin{array}{l}760 \\
1610\end{array}$ & $\begin{array}{l}<0.09 \\
<0.06\end{array}$ & $\begin{array}{l}<270 \\
<600\end{array}$ & 250 & $100-1500$ \\
\hline $\begin{array}{l}\zeta \mathrm{Oph}^{i} \\
\zeta \mathrm{Oph}^{j}\end{array}$ & $\begin{array}{l}2.78 \pm .01 \\
2.85 \pm .05\end{array}$ & $\begin{array}{l}0.89 \pm .06 \\
0.67,0.44\end{array}$ & $\begin{array}{l}2.84(12) \\
2.88(12)\end{array}$ & $\begin{array}{l}\leq 3 \\
\leq 3\end{array}$ & $\begin{array}{l}\cdots \\
\ldots\end{array}$ & $\begin{array}{l}\cdots \\
\cdots\end{array}$ & $\begin{array}{l}7,8,9 \\
7,8,9\end{array}$ & $\begin{array}{l}30 \\
30\end{array}$ & $\begin{array}{l}2.0(-4) \\
2.0(-4)\end{array}$ & $\begin{array}{l}0.057 \\
0.13\end{array}$ & $\begin{array}{l}289 \\
660\end{array}$ & $\begin{array}{l}<0.07 \\
<0.10\end{array}$ & $\begin{array}{l}<355 \\
<508\end{array}$ & $\begin{array}{l}150 \\
150\end{array}$ & $\begin{array}{l}\leq 200 \\
\leq 200\end{array}$ \\
\hline HD $154368^{i}$ & 2.87 & 0.65 & $2.75(13)$ & $19 \pm 5$ & $0.4-1.0$ & $\ldots$ & 2,10 & 25 & $\begin{array}{l}4.0(-4) \\
1.0(-4)\end{array}$ & $\begin{array}{l}0.15 \\
0.10\end{array}$ & $\begin{array}{l}375 \\
1000\end{array}$ & $\begin{array}{l}0.086 \\
0.047\end{array}$ & $\begin{array}{l}215 \\
466\end{array}$ & 250 & $200-2000$ \\
\hline HD $154368^{j}$ & 3.03 & 0.65 & $2.48(13)$ & $19 \pm 5$ & $0.4-1.0$ & $\cdots$ & 2,10 & 25 & $\begin{array}{l}4.0(-4) \\
1.0(-4)\end{array}$ & $\begin{array}{l}\cdots \\
\cdots\end{array}$ & $\begin{array}{l}\ldots \\
\ldots\end{array}$ & $\begin{array}{l}0.078 \\
0.043\end{array}$ & $\begin{array}{l}194 \\
425\end{array}$ & 250 & $200-2000$ \\
\hline HD 169454 & $2.9_{-.4}^{+.6}$ & $0.3-0.5$ & see text & $<24$ & 0.46 & $3.38(13)$ & $2,6,11$ & 15 & $\begin{array}{l}7.1(-5) \\
4.3(-5)\end{array}$ & $\begin{array}{l}0.12 \\
0.077\end{array}$ & $\begin{array}{l}1680 \\
1780\end{array}$ & $\begin{array}{l}0.018^{k} \\
0.016^{k}\end{array}$ & $\begin{array}{l}250^{k} \\
350^{k}\end{array}$ & 300 & $200-5000$ \\
\hline HD 210121 & $2.8 \pm .2$ & $1.0:$ & $1.2(13)$ & $\leq 27$ & 1.02 & $1.23(13)$ & $2,6,12$ & 20 & $\begin{array}{l}1.0(-4) \\
6.7(-5) \\
3.4(-5)\end{array}$ & $\begin{array}{l}0.10 \\
0.08 \\
0.052 \\
\end{array}$ & $\begin{array}{l}1000 \\
1200 \\
1530 \\
\end{array}$ & $\begin{array}{l}\leq 0.06 \\
\leq 0.048 \\
\leq 0.029\end{array}$ & $\begin{array}{l}\leq 600 \\
\leq 700 \\
\leq 850 \\
\end{array}$ & 300 & $1000-8000$ \\
\hline
\end{tabular}

${ }^{a}$ From measurements of $\mathrm{C}_{2}$ excitation where available; estimated otherwise.

${ }^{b}$ From detailed cloud models ( $\zeta$ Oph, $\zeta$ Per, HD 169454, HD 210121) or estimated.

' From measured $T_{01}$; see col. (2).

d From limit on or measurement of the $\mathrm{CN} 113.491 \mathrm{GHz}$ emission line.

e From analysis of $\mathrm{C}_{2}$ excitation.

f From analysis of ${ }^{12} \mathrm{CO}$ emission-line ratios.

8 Analysis of data of Cardelli et al. 1990.

b Preliminary analysis of data of Gredel et al. 1991a.

i For the fit of a single velocity component to the $\mathrm{CN}$ data.

j For the fit of two velocity components, assumed to have equal values of $T_{01}$.

k For models 1 and 2 of Jannuzi et al. 1988. The limit on $T_{\mathrm{R}}$ suggests $n(e)<0.025 \mathrm{~cm}^{-3}$ in either case.

ReFERENCES.- (1) Meyer et al. 1989; (2) van Dishoeck et al. 1991; (3) Kaiser \& Wright 1990; (4) Crawford 1990; (5) Cardelli et al. 1990; (6) Gredel et al. 1991a; (7) Crane et al. 1986 and Crane et al. 1989; (8) Lambert et al. 1990; (9) Black \& van Dishoeck 1988; (10) Palazzi et al. 1990; (11) Jannuzi et al. 1988; (12) Gredel et al. $1991 b$.

techniques, such as the optical absorption lines of $\mathrm{C}_{2}$ (van Dishoeck \& Black 1989; Gredel, van Dishoeck, \& Black 1991a), and the CO emission-line ratios (van Dishoeck et al. 1991).

The derived electron densities are typically of the order of $n(e) \approx 0.1 \mathrm{~cm}^{-3}$. The corresponding neutral densities are generally in harmony with the ranges of density inferred from the excitation of $\mathrm{CO}$ rotational lines and favor in most cases the lower densities. They are usually somewhat larger than those indicated by the excitation of $\mathrm{C}_{2}$, except for the thinnest clouds toward $\zeta$ Oph and $\zeta$ Per. The different densities inferred from $\mathrm{C}_{2}$ and $\mathrm{CN}$ may reflect chemical stratification: $\mathrm{CN}$ may be most highly concentrated in the densest parts of a cloud while $\mathrm{C}_{2}$ is more broadly distributed. This possibility has been explored in chemical models by van Dishoeck et al. (1991), but the difference in column-averaged densities is found to be small. Alternatively, the effect may point to lingering uncertainties about the collisional excitation cross sections for $\mathrm{C}_{2}$. The low limit on $T_{R}(113.49)$ and the well-constrained values of $T_{01}$ and $N(\mathrm{CN})$ toward $\zeta$ Oph exclude models of high density (Viala, Roueff, \& Abgrall 1988) that have been invoked to explain other observations of this cloud (cf. also Black 1988 and van Dishoeck et al. 1991).

The CN optical absorption and millimeter emission lines yield densities that generally agree within a factor of 2 , which is well within the range allowed by the uncertainties. This agreement suggests that the density does not vary drastically over the large range of angular scales, $10^{-3}$ to $60^{\prime \prime}$, sampled by the two techniques. However, the two methods are not equally sensitive: the $R(1) / P(1)$ equivalent width ratio is often sufficiently uncertain that it allows a range of $b$ values too large to constrain the curve of growth (see the example of HD 169454, in $\S 3$ ). Only if the $N=0$ column densities are determined independently from unsaturated lines of the $A^{2} \Pi-X^{2} \Sigma^{+}$red system (van Dishoeck and Black 1989; Gredel et al. 1991a) or of the $B-X(1,0)$ band (Meyer \& Roth 1990) can an accurate value of $T_{01}$ be found.

The cloud toward HD 62542 is a noteworthy example. Cardelli et al. (1990) report values of $T_{01}=3.7 \pm 0.9 \mathrm{~K}(2 \sigma)$, $N(\mathrm{CN})=2.6 \times 10^{13} \mathrm{~cm}^{-2}$, and $b=0.79 \mathrm{~km} \mathrm{~s}^{-1}$ from a curveof-growth fit to $B-X(0,0)$ observations. Our reanalysis including spin-rotation fine structure yields slightly different results 
for the same data (see Table 1). The nominal value of the excitation temperature implies high densities $n(e) \approx 0.1-0.5$ $\mathrm{cm}^{-3}$ and $n\left(\mathrm{H}_{2}\right) \approx 1500-4000 \mathrm{~cm}^{-3}$ for the range of fractional ionizations considered. Consequently, the millimeter emission lines of $\mathrm{CN}$ are predicted to be strong, $\int T_{R}(113.49) d V \approx$ $0.3-0.4 \mathrm{~K} \mathrm{~km} \mathrm{~s}^{-1}$. Independent observations by Gredel et al. (1991a) of both the $B-X(0,0)$ and $A-X(2,0)$ bands yield somewhat lower values of $T_{01}$ and $b$ and higher values of $N(\mathrm{CN})$, although the results agree with those of Cardelli et al. within the combined uncertainties. On the basis of the nominal values from the Gredel et al. data, we find $n(e) \approx 0.005-0.05$ $\mathrm{cm}^{-3}$ and $n\left(\mathrm{H}_{2}\right) \approx 150 \mathrm{~cm}^{-3}$ and predict $\int T_{R}(113.49) d V \approx$ $0.025-0.050 \mathrm{~K} \mathrm{~km} \mathrm{~s}^{-1}$. This illustrates how a $15 \%$ difference in derived values of $T_{01}$ translates into an order-of-magnitude difference in derived densities. In such a case, measurements of the millimeter emission lines, combined with the optically determined column density, provide much stronger constraints on density. A recent search for $\mathrm{CN}$ emission toward HD 62542 reveals only weak lines, $\int T_{R}(113.49) d V \approx 0.12 \mathrm{~K} \mathrm{~km} \mathrm{~s}^{-1}$ (Gredel et al. 1991a) and suggests $n(e) \approx 0.03-0.13$ and $n\left(\mathrm{H}_{2}\right) \approx 400-750 \mathrm{~cm}^{-3}$. This conclusion would not be seriously modified if the star is not located fully behind the emitting molecules, because the weak $113.49 \mathrm{GHz}$ line is already partly saturated; however, the effect might be noticed in hyperfine line ratios at higher signal-to-noise ratio.

The fractional ionization $n(e) / n\left(\mathrm{H}_{2}\right)$ is an important parameter in the analysis. Except in well-studied diffuse clouds, $n(e) / n\left(\mathrm{H}_{2}\right)$ is not currently determined independently. At most, the value would be comparable to the abundance of carbon that is not in the form of $\mathrm{CO}$ or dust. Models suggest that ultraviolet photoionization remains important inside translucent clouds and that plausible values are $n(e) / n\left(\mathrm{H}_{2}\right) \approx 3$ $\times 10^{-5}$ to $3 \times 10^{-4}$ (van Dishoeck \& Black 1989). Even in the extreme limits, $n(e) / n\left(\mathrm{H}_{2}\right) \sim 0$ and $\sim \infty$, the derived densities are altered by factors of 2 at most. In principle, the $\mathrm{CN}$ observations could be used to determine both $n(e)$ and $n(e) / n\left(\mathrm{H}_{2}\right)$, if the $\mathrm{H}_{2}$ impact rates were reliably known. The electron collisions obey a strong selection rule, $\Delta N= \pm 1$, while collisions with $\mathrm{H}_{2}$ should induce, and perhaps favor, transitions with $|\Delta N|>1$. As a result, the observable intensity ratio of the 226.875 and $113.491 \mathrm{GHz}$ lines will be sensitive to the electron fraction. In practice, this line ratio is about 0.2 and varies by only $20 \%$ for $n(e) / n\left(\mathrm{H}_{2}\right) \approx 3 \times 10^{-5}$ to $3 \times 10^{-4}$, which is probably not accurately measurable for weak lines. The lack of $\mathrm{H}_{2}-\mathrm{CN}$ collision rates also limits the accuracy of derived electron densities. The derived electron densities scale with the neutral collision rate in the same manner as they vary with $n\left(\mathrm{H}_{2}\right) / n(e)$. As seen in Table $1, n(e)$ typically varies by a factor of 3-4 when $n(e) / n\left(\mathrm{H}_{2}\right)$ ranges over a factor of 10 .

Other polar molecules could be used similarly to infer electron densities. The CS molecule has an even larger dipole moment than $\mathrm{CN}$. CS has a strong absorption system in the near-ultraviołet, and CS millimeter emission lines have already been detected in some of the same clouds discussed here (Drdla, Knapp, \& van Dishoeck 1989). Observations of CS and $\mathrm{CN}$ in the same cloud could be used to determine both $n(e)$ and $n(e) / n\left(\mathrm{H}_{2}\right)$, but the near-ultraviolet absorption-line measurements will be crucial to provide an independent determination of the CS column density.

In summary, we have shown that existing data on emission and absorption lines of $\mathrm{CN}$ can be used to estimate electron densities in diffuse and translucent clouds. Derived densities lie in the range $n(e) \approx 0.02-0.5 \mathrm{~cm}^{-3}$. There is good agreement with neutral densities derived by other techniques, although the CN measurements generally favor the lower values. Eventually, the combination of the absorption and millimeter data might lead to a determination of the electron fraction as a function of cloud thickness, $A_{V}$. This would be important not only for testing theoretical models but also for insight into the coupling of magnetic fields and cloud structure.

J. H. B. is grateful to the Swedish Natural Science Research Council and NORDITA for financial assistance. This work was partly supported by NASA through grant NAGW-763 to the University of Arizona, and grant NAGW-1945 to the California Institute of Technology.

\section{REFERENCES}

Allison, A. C., \& Dalgarno, A. 1971, A\&A, 13, 331

Bauschlicher, C. W., Jr., Langhoff, S. R., \& Taylor, P. R. 1988, ApJ, 332, 531

Black, J. H. 1988, Adv. Atom. Mol. Phys., 25, 477

Black, J. H., \& van Dishoeck, E. F. 1988, ApJ, 331, 986

Cardelli, J. A., Suntzeff, N. B., Edgar, R. J., \& Savage, B. D. 1990, ApJ, 362, 551

Crane, P., Hegyi, D. J., Kutner, M. L., \& Mandolesi, N. 1989, ApJ, 346, 146

Crane, P., Hegyi, D. J., Mandolesi, N., \& Danks, A. C. 1986, ApJ, 309, 822

Crawford, I. A. 1990, MNRAS, 243, 593

Dickinson, A. S., Phillips, T. G., Goldsmith, P. F., Percival, I. C., \& Richards, D. 1977, A\&A, 54, 645

Drdla, K., Knapp, G. R., \& van Dishoeck, E. F. 1989, ApJ, 345, 815

Field, G. B., \& Hitchcock, J. L. 1966, Phys. Rev. Letters, 16, 817

Gredel, R., van Dishoeck, E. F., and Black, J. H. 1991a, in preparation

Gredel, R., van Dishoeck, E. F., de Vries, C. P., \& Black, J. H. 1991b, A\&A, submitted

Green, S., \& Chapman, S. 1978, ApJS, 37, 168

Jannuzi, B. T., Black, J. H., Lada, C. J., \& van Dishoeck, E. F. 1988, ApJ, 332, 995

Kaiser, M. E., \& Wright, E. L. 1990, ApJ, 356, L1
Lambert, D. L., Sheffer, V., \& Crane, P. 1990, ApJ, 359, L19

Lepp, S., Dalgarno, A., van Dishoeck, E. F., \& Black, J. H. 1988, ApJ, 329, 418

Mather, J. C., et al. 1990, ApJ, 354, L37

Meyer, D. M., \& Jura, M. 1985, ApJ, 297, 119

Meyer, D. M., \& Roth, K. C. 1990, ApJ, 349, 91

Meyer, D. M., Roth, K. C., \& Hawkins, I. 1989, ApJ, 343, L1

Palazzi, E., Mandolesi, N., Crane, P., Kutner, M. L., Blades, J. C., \& Hegyi, D. J. 1990, ApJ, 357, 14

Shklovsky, I. S. 1966, Astron. Tsirk., No. 364, p.

Skatrud, D. D., DeLucia, F. C., Blake, G. A., \& Sastry, K. V. L. N. 1983, J. Molec. Spectrosc., 99, 35

Thaddeus, P. 1972, ARA\&A, 10, 305

Thaddeus, P., \& Clauser, J. F. 1966, Phys. Rev. Letters, 16, 819

Thompson, R., \& Dalby, F. W. 1968, Canadian J. Phys., 46, 2815

van Dishoeck, E. F., \& Black, J. H. 1986, ApJS, 62, 109

- 1989, ApJ, 340, 273

van Dishoeck, E. F., Black, J. H., Phillips, T. G., \& Gredel, R. 1991, ApJ, 366, 141

Viala, Y., Roueff, E., \& Abgrall, H. 1988, A\&A, 190, 215 